\title{
GEOMETRIA DE GREENSTONE BELTS ARQUEANOS DA REGIÃO DE RIO MARIA (SUDESTE DO PARÁ, BRASIL), A PARTIR DE INTERPRETAÇÃO GRAVIMÉTRICA
}

\author{
ZORANO S. SOUZA*, JOSÉ G. LUIZ**, JOÃO C.R. CRUZ ${ }^{* * *}$ e REINALDO N. PAIVA*****
}

\begin{abstract}
GEOMETRY OF ARCHEAN GREENSTONE BELTS OF THE RIO MARIA REGION (SOUTHEAST PARÁ, BRAZIL) FROM GRAVIMETRIC INTERPRETATION. The aim of this paper is to discuss the geometric and tectonic aspects of the granite-greenstone terrain located in the Rio Maria region, SE Pará, Brazil. It is based on the correlation of surface geological data and a gravity profile observed between Rio Maria and Xinguara towns. The gravity profile shows two highs related to the Serrinha and Identidade greenstone belts. The former has a flat shape, while the later one behaves as an assymetric synformal body with southern its flank plunging slightly toward the north. The continuity between these two bodies suggests that, originally, the Serrinha and Identidade belts were connected and belonged to a single unit that was subsequently divided by the forceful intrusion of the surrounding granitoids. The gneissic terrains observed in the northern part of the Identidade area owe their geometry, probably to listric faults reaching the base of the continental crust The geometric and tectonic constructions show that the two greenstone belts can be figured by different two-dimensional shapes, but they are restricted to the shallow part of the continental crust; their depth never exceeds $5 \mathrm{~km}$. On the other hand, the thickness of the late Archean crust reached at least $18 \mathrm{~km}$ at the time of the metamorphic event
\end{abstract}

Keywords: Greenstone belts, granites, Archean, SE of Pará State, gravimetry, tectonic.

RESUMO Este artigo discute alguns aspectos geométricos e tectônicos do terreno granito-greenstone da região de Rio Maria, sudeste do Estado do Pará, Brasil. Baseia-se em um perfil gravimétrico e em dados geológicos obtidos entre as cidades de Rio Maria e Xinguara. No perfil gravimétrico distinguem-se dois altos, relacionados aos greenstone belts de Serrinha e Identidade. O primeiro tem forma aproximadamente tabular suborizontal, enquanto o ultimo dispõe-se como um sinforme assimétrico, cujo flanco sul mergulha suavemente para none. O modelamento geométrico dessas faixas sugere que elas formavam um único corpo, o qual foi seccionado posteriormente pelas intrusões dos granitóides. Os terrenos gnáissicos aflorantes a norte da Faixa Identidade provavelmente adquiriram a presente configuração através de falhas lístricas existentes na base da crosta continental. A reconstrução geométrica e tectônica mostra que os greenstone belts em análise podem ter formas bi-dimensionais variadas. Porém, eles se restringem às partes mais superficiais da crosta, não ultrapassando profundidades maiores que $5 \mathrm{~km}$. A espessura da crosta seria de pelo menos 18 $\mathrm{km}$ ao tempo do evento metamórfico do final do Arqueano.

Palavras-chaves: Greenstone belts, granites, Arqueano, SE do Estado do Pará, gravimetria, tectônica.

INTRODUÇÃO A aplicação de métodos geofísicos de grande escala aos terrenos granito-greenstone foram enfatizados apenas a partir de meados dos anos 70 (síntese de Condie 1981, p. 10-12). Recentemente, uma série de workshops promovidos pelo Canadian Continental Drilling Program (CCDP) e Geological Survey of Canada, reavaliaram novamente este tema. Um resumo das principais conclusões destes encontros deve-se a Hall \& Drury (1989), sendo referidas nos parágrafos abaixo.

As interpretações prévias consideravam os greenstone belts com formas sinclinoriais e o diapirismo granítico como os causadores do padrão estrutural. Atualmente, os greenstone belts são interpretados como faixas de geometrias variadas (salsichas, cônicas, tubulares) que sofreram colagens tectônicas e pelo menos um período de transpressão, tendo o regime tangencial afetado também os granitóides adjacentes. As espessuras e profundidades aparentes, fornecidas pelos métodos geofísicos (gravimetria, sísmica de refração, sísmica de reflexão, fluxo térmico), variam de 1 a $3 \mathrm{~km}$ em Yellowknife, cerca de $5 \mathrm{~km}$ no Abitibi, 6 a $8 \mathrm{~km}$ em Wabigopn e 3 a $7 \mathrm{~km}$ em Barberton. Por outro lado, as espessuras inferidas pelos levantamentos geológicos variam de 6 a $15 \mathrm{~km}$. No Wabigoon, os granitóides alcançam usualmente $7 \mathrm{~km}$ de profundidade (podendo ter raizes até $11 \mathrm{~km}$ ), com alguns corpos atingindo somente de 2 a $3 \mathrm{~km}$.
Os modelamentos geofísicos indicam que os greenstone belts apresentam pequena extensão vertical (limitam-se aos primeiros $16-20 \mathrm{~km}$ da crosta continental) e constituem estruturas assimétricas. Nestas profundidades, existem refletores sísmicos contínuos abaixo de ambos granitóides e green-stone belts. Tal fato sugere que as litologias subjacentes (crosta intermediária) estão separadas do bloco tectônico granito-greenstone (Condie 1981, Beer et al. 1986, Thomas et al. 1986, Hall \& Drury 1989). As grandes descontinuidades estruturais encontradas no interior dos greenstone belts e nos contatos destes com os granitóides e/ou terrenos gnáissicos seriam, na verdade, falhas lístricas transformadas em zonas de deslocamento a 10-15 km de profundidade (Hall \& Drury 1989).

$\mathrm{Na}$ região central do Brasil, o Projeto Geofísico BrasilCanadá (DNPM1979) definiu curvas de anomalias magnéticas de campo total de direções WNW-ESE e E-W. Interpretando dados gravimétricos e aeromagnéticos, Hasui et al. (1984) tentaram dividir o Cráton Amazônico em blocos tectônicos, estando a área de interesse inserida no chamado Bloco Araguacema. Em trabalho recente, Carvalho (1988) demonstrou que as isoanomalias Bouguer sofrem nítida inflexão quando passam do Cráton Amazônico (onde têm direções WNW-ESE ou E-W) para a Faixa de Dobramentos Araguaia (onde são N-S). Tais informações permitem separar estas duas grandes unidades

* Departamento de Geologia, Universidade Federal do Rio Grande do Norte, Caixa Postal 1502, CEP 59072-970, Natal, RN, Brasil

** Departamento de Geofísica, Universidade Federal do Pará, Caixa Postal 1611, CEP 66059-000, Belém, PA, Brasil

*** Curso de Pós-Graduação em Geofísica, Universidade Federal do Pará, Caixa Postal 1611, CEP 66059-000, Belém, PA, Brasil

**** Conpetro Geofísica S/A, Rio de Janeiro, Brasil 
tectônicas, mas são insuficientes na distinção de unidades menores internas ao cráton (Silva \& Sá 1982, Almeida 1985, Almeida et al. 1986, Carvalho 1988). Especificamente para a região de Rio Maria, Carvalho (1989) descreveu importantes anomalias Bouguer relacionadas às áreas de ocorrência dos greenstone belts de Serrinha e Identidade.

Este trabalho analisa dados gravimétrícos referentes a região de Rio Maria (Fig. 1). Eles resultaram do levantamento realizado por estudantes do Curso de Mestrado em Geofísica do Centro de Geociências da Universidade Federal do Pará (Cruz \& Paiva 1988). Pretende-se, então, fazer a correlação entre os modelos obtidos pelas interpretações gravimétricas e aqueles sugeridos pela geologia de superfície, confrontando-os com os resultados conhecidos para os terrenos arqueanos de outras partes do mundo.

GEOLOGIA DA ÁREA A região de Rio Maria localiza-se no extremo sudeste do Estado do Pará, $200 \mathrm{~km}$ a sul da Serra dos Carajás. Em termos regionais, ela compreende a borda leste do Cráton Amazônico (na denominada Província Tapajós de Almeida et al. 1977), próximo ao limite com a Faixa de Dobramentos Araguaia. Sínteses sobre a geologia regional podem ser encontradas em Hirata et al (1982), Amaral (1984), Santos \& Loguércio (1984) e DOCEGEO (1988). Artigos mais específicos sobre a área de interesse são referidos a Medeiros et al (1987), Huhn et al (1988) e Souza et al $(1988,1990)$.

A figura 1 contém o mapa geológico e uma coluna litoestratigráfica da área, tendo sido parcialmente modificados daqueles apresentados por DOCEGEO (1988) e Huhn et al (1988). Sucintamente, reconhecem-se as seguintes unidades: 1. terrenos gnáissicos, de composição tonalítica a granodiorítica, constituindo o embasamento regional (Complexo Xingu), aflorantes nas proximidades de Xinguara. Gnaisses similares, $55 \mathrm{~km}$ a sul de Rio Maria, possuem idades de $2800 \pm 8 \mathrm{Ma}$ $(\mathrm{U} / \mathrm{Pb})$ e $2605 \pm 32 \mathrm{Ma}(\mathrm{Rb} / \mathrm{Sr})$ (Macambira et al 1990);

2. faixas de greenstone belts (Supergrupo Andorinhas de DOCEGEO 1988), compostos predominantemente por metamáficas (basaltos, gabros) e metaultramáficas, com proporções subordinadas de metadacitos, metassiltitos e metagrauvacas, e raras lentes de formação ferrífera bandada. Neste trabalho serão enfatizadas as seqüências Serrinha e Identidade (localização na Fig. 1), constituídas essencialmente por rochas meta vulcânicas;

3. metagranitóides variegados, intrusivos nos greenstone belts, compostos de granodioritos com abundantes enclaves máficos, quartzodioritos, trondhjemitos, tonalitos e leucogranitos, todos com idades $\mathrm{Rb} / \mathrm{Sr}$ no intervalo de $2660 \pm 60 \mathrm{Ma}$ a $2528 \pm 35$ Ma (Montalvão et al 1984,1988, Dall'Agnol et al 1986, Gastai et al 1987, Macambira et al 1990);

4. coberturas plataformais contendo sedimentos pelíticos (folhelhos), elásticos (arenitos, conglomerados) e químicos (cherts, dolomites), anquimetamórficos, correlacionados ao Grupo Rio Fresco (Hirata et al 1982, DOCEGEO 1988, Huhn et al 1988);

5. diques ácidos, correlacionados ao magmatismo granítico anorogênico do Proterozóico Médio, cujos maciços afloram expressivamente a sul de Rio Maria (Dall'Agnol et al 1986, Medeiros et al 1987, DOCEGEO 1988, Souza et al 1990).

\section{ESTRUTURAÇÃO DAS UNIDADES ARQUEANAS}

Aspectos estruturais da região de Rio Maria foram discutidos por Medeiros et al (1987), Huhn et al (1988) e Souza et al (1988, 1990). Sumariamente (Souza et al 1988, 1990), tem-se o embasamento gnáissico (Complexo Xingu) com direções tectônicas (foliação, bandamento metamórfico) orientadas NW-SE, com mergulhos moderados a fortes para SW. Os greenstone belts e metagranitóides arqueanos possuem direções estruturais similares, embora menos proeminentes, sendo mais marcantes em zonas de cisalhamento dúctil.
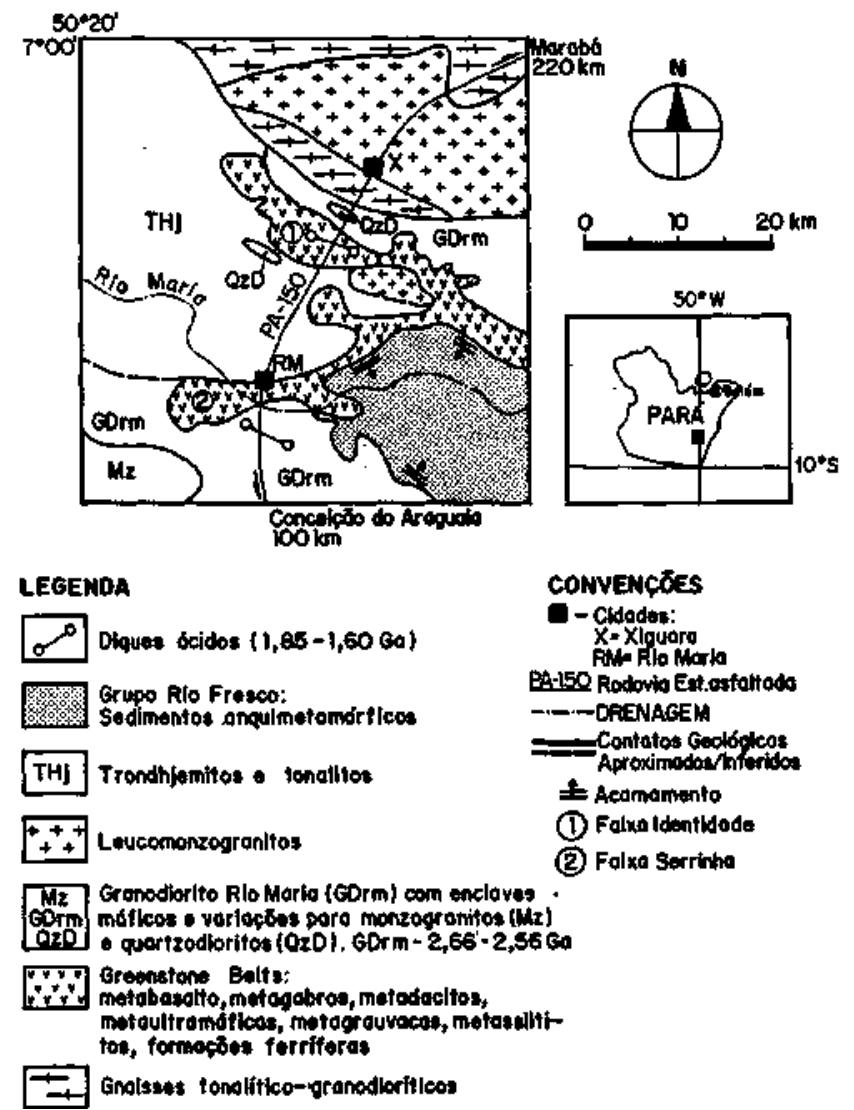

Figura 1 - Mapa geológico da região de Rio Maria (modificado de DOCEGEO 1988 e Huhn et al. 1988)

Figure 1 - Geologic map of the Rio Maria region (adapted from DOCEGEO 1988 and Huhn et al. 1988)

O relacionamento tectônico entre os conjuntos litoestratigráficos arqueanos ainda não foi devidamente estabelecido, existindo três possibilidades tal como mostra a figura 2 (compilada de Z.S. Souza, tese de doutorado em andamento CPGG/UFPA). De acordo com esta figura, os greenstone belts teriam sido comprimidos entre dois blocos de granitóides num regime de transpressão dextrógira, resultando na formação de estruturas em flor positiva. As diferenças básicas entre os casos $\mathrm{a}, \mathrm{b}$ e c da figura 2 referem-se à extensão e forma, em profundidade, dos greenstone belts, distinguindo-se os casos sem raiz (Fig. 2a) e com raiz (Figs. 2b e 2c).

LEVANTAMENTO GRAVIMÉTRICO Os dados gravimétricos analisados neste trabalho foram obtidos ao longo da rodovia PA-150, partindo-se de um ponto $7 \mathrm{~km}$ a sul de Rio Maria e terminando a $1 \mathrm{~km}$ a norte de Xinguara, perfazendo um total de $37 \mathrm{~km}$. As medições foram feitas a intervalos de 1 $\mathrm{km}$ com um gravímetro LaCoste-Romberg, usando-se como base de referência o marco altimétrico do IBGE (coordenadas $7^{\circ} 18^{\prime} 39^{\prime \prime}$ S e $\left.50^{\circ} 02^{\prime} 49^{\prime \prime} \mathrm{W}\right)$, localizado na cidade de Rio Maria. Posteriormente, essa base e todos os dados foram referidos à base gravimétrica denominada DOCEGEO/RIO MARIA, localizada nas coordenadas $7^{\circ} 16^{\prime} 18^{\prime \prime} \mathrm{S}$ e $50^{\circ} 05^{\prime} 48^{\prime \prime} \mathrm{W}$, cujo valor no sistema GRS-67 é 978044,87 mGal (Carvalho 1988).

AVALIAÇ̃̃O DE DENSIDADES As densidades aqui utilizadas (Tab. 1) foram compiladas de Z. S. Souza (tese de doutorado em andamento, CPGG/UFPA). Usaram-se amostras de rochas frescas das litologias representativas do mapeamento geológico (Fig. 1), comparando-se os tipos preservados e seus equivalentes deformados (séries miloníticas de metabasaltos, metagabros, metaultramáficas, quartzodioritos, trondhjemitos 
(a)

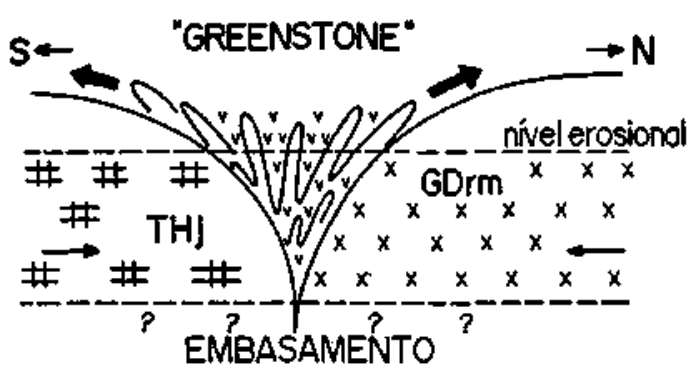

(b)

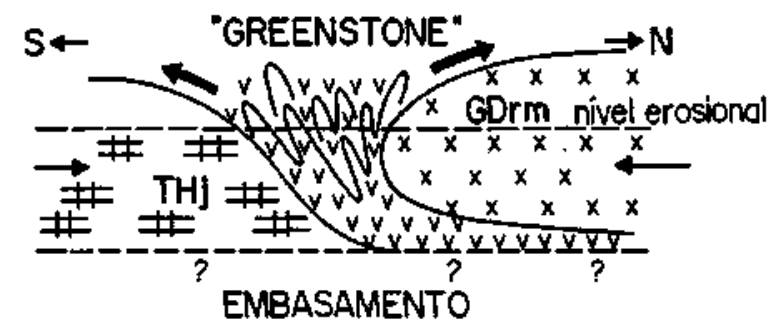

(c)

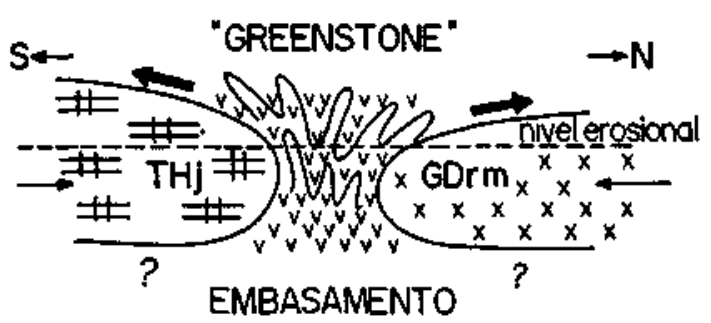

Figura 2 - Perfis esquemáticos de direção $S$ - $N$, mostrando as possíveis relações geométricas entre os granitóides e os greenstone belts: $\boldsymbol{a}$, greenstone sem raiz; $\boldsymbol{b}$. e c . greenstone com raiz. GDrm = granodiorito Rio Maria, THJ = trondhjemitos. As setas fina e espessa indicam, respectivamente, as direções de compressão e fuga de material

Figure 2 - Schematic S-N profiles showing the possible geometric relations between the granitoid bodies and greenstone belts: a. unrooted greenstone; $\mathbf{b}$. and $\mathbf{c}$. rooted greenstone. GDrm = Rio Maria granodiorite, $\mathrm{THJ}=$ trondhjemites. The thin and thick arrows indicate, respectivelly, compression and material transfer directions

e gnaisses do Complexo Xingu). Para as determinações de densidades, usou-se o método clássico de pesar a amostra no ar e dentro d'água. Admitindo-se uma densidade média de 1 $\mathrm{g} / \mathrm{cm}^{3}$ para a água, o peso da água deslocada pela amostra corresponde ao volume desta.

Em função do predomínio de determinadas litologias e pelo fato de que as variações de densidade entre as rochas preservadas e os seus equivalentes miloníticos são, em geral, muito pequenas (Tab. 1), foram utilizados os seguintes intervalos de densidades: metabasaltos, metagabros e metaultramáficas $2,95 \mathrm{~g} / \mathrm{cm}^{3}$ a $3,02 \mathrm{~g} / \mathrm{cm}^{3}$; metadacitos, granodioritos, trondhjemitos, tonalitos e gnaisses $-2,63 \mathrm{~g} / \mathrm{cm}^{3}$ a $2,77 \mathrm{~g} / \mathrm{cm}^{3}$.

INTERPRETAÇÃO GRAVIMÉTRICA Nos modelos utilizados, foram consideradas as fontes das anomalias como polígonos fechados dispostos perpendicularmente ao perfil realizado. Tal suposição é corroborada pela geologia de superfície e pelas orientações WNW-EWE (na Faixa Identidade) e E-W (na Faixa Serrinha) das isoanomalias definidas por Carvalho $(1988,1989)$. O cálculo do efeito gravimétrico produzido por estas fontes foi obtido por intermédio de um programa computacional escrito para o algoritmo de Talwani et al. (1959) para o caso 2D, e modificado para 2,5D por Cady (1977). Os valores calculados e observados das anomalias gravimétricas foram comparados visualmente. Sempre que uma grande discrepância entre esses valores era encontrada, modificava-se o
Tabela 1 - Valores de densidades medidas para os litotipos presentes na área pesquisada

Table 1 - Measured density values for the lithologies found in the studied area

\begin{tabular}{l|c|l}
\hline LITOLOGIAS & $\mathrm{g} / \mathrm{cm}^{3}$ & \multicolumn{1}{|c}{ OBSERVAC̄́ES } \\
\hline Metadacitos & $2,65-2,68$ & Tipo preservado - Protomilonito \\
& 2,64 & Ultramilon. macico, c/sulfetos \\
& 2,58 & Blastomilonito micáceo \\
\hline Metagabros & 3,03 & Tipo preservado, c/sulfetos \\
& 2,93 & Protomilonito xistoso \\
\hline & $3,02-3,05$ & Tipo preservado, macico \\
Metabasaltos & 3,18 & Blastomilon. macico, c/sulfetos \\
& 2,85 & Blastomilonito xistoso \\
\hline Metaultramáficas & $2,81 \cdot 2,97$ & Tremolita-talco-clorita xisto \\
\hline Leucogranitóides & 2,30 & Filonito (talco-clorita) alterado \\
\hline Trondhjemitos & 2,68 & Tipos pouco deformados \\
\hline Granodionilon. macico, c/sulfetos \\
Rio Maria & $2,63-2,70$ & Blastomilon. macico, c/sulfetos \\
\hline Quartzodionitos e & $2,83 \cdot 2,89$ & Tipos preserv. - ultramilonitos \\
\hline enclaves máficos & 2,77 & Tipos preservados - milonitos \\
\hline Gnaisses & 2,63 & Tipos tonalíticos \\
\hline
\end{tabular}

modelo, tentando obter-se, por tentativa, uma resposta mais próxima dos dados observados. Durante este processo de modelagem, usaram-se como vínculos as densidades medidas e a extensão em superfície das unidades mapeadas. Como primeira aproximação, partiu-se dos modelos geológicos da figura 2 , resultando nas três possibilidades discutidas nos parágrafos seguintes.

As figuras $3 \mathrm{a}, 3 \mathrm{~b}$ e $3 \mathrm{c}$ representam os modelos deduzidos a partir dos dados gravimétricos. As anomalias Bouguer encontradas permitem definir dois picos, sendo o de menor intensidade na Faixa Serrinha e o de maior expressão na Faixa Identidade. Geologicamente, observa-se que, em confronto com os granitóides e metadacitos, as rochas máficas e ultramáficas dos greenstone belts fornecem anomalias relativas positivas de cerca de $13 \mathrm{mGal}$ na Faixa Serrinha e de $26 \mathrm{mGal}$ a $29 \mathrm{mGal}$ na Faixa Identidade.

Nos modelos 1 (Fig. 3a), 2 (Fig. 3b) e 3 (Fig. 3c), a Faixa Serrinha (localizada entre as estações 1 e 11) apresenta uma forma tabular, suborizontal, contínua (modelo 1) ou descontínua (modelos 2 e 3). A extensão aflorante (entre as estações 6 e 9) corresponde à espessura aparente avaliada na geologia de superfície. A parte não aflorante do corpo central mostra que o greenstone belt se prolonga sob os granitóides, extendendo-se inclusive para sul da estação 1 (modelos 2 e 3 ). A profundidade máxima do pacote supracrustal seria de $1,8 \mathrm{~km}$.

Já, a Faixa Identidade (localizada entre as estações 17 e 29) apresenta variações nos três resultados teóricos. No primeiro (Fig. 3a), aparece um corpo tabular, raso, vertical, com $0,7 \mathrm{~km}$ de espessura (em torno da estação 23 ), bem como um corpo maior sinformal, assimétrico na sua parte superior e tabular, suborizontal (profundidade de $6,6 \mathrm{~km}$ ), na sua parte inferior. O modelo 2 (Fig. 3b) apresenta semelhanças com o anterior, diferindo por ter o corpo maior uma geometria sinformal típica, com menor assimetria e atingindo a profundidade de 7,6 $\mathrm{km}$. O modelo 3 (Fig. 3c) mostra diferenças importantes em relação aos anteriores: 1. o corpo menor, que parecia tabular e vertical nos casos 1 e 2, agora é complexo e tem maior área de exposição em superfície, com profundidade máxima de 1,4 $\mathrm{km} ; \mathbf{2}$. o corpo mais volumoso tem geometria sinformal bas- 

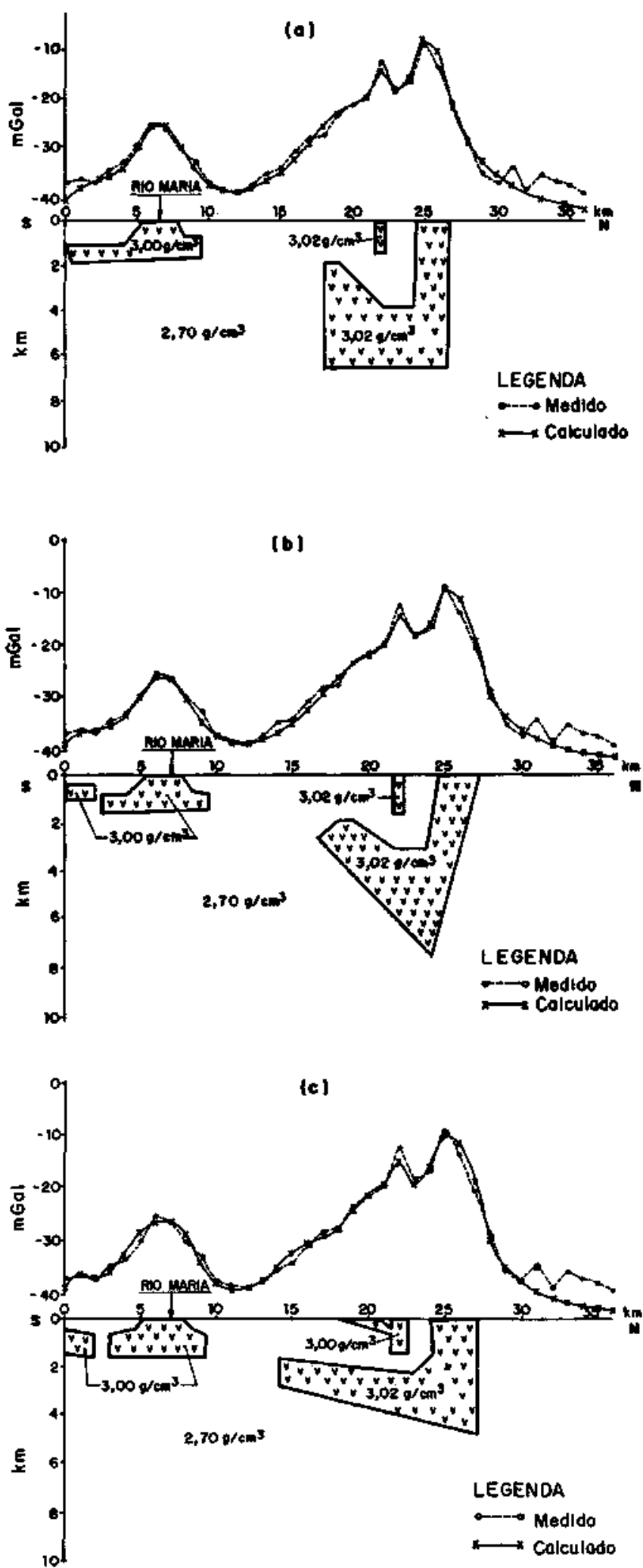

Figura 3 - Modelos gravimétricos bi-dimensionais para o perfil Rio Maria-Xinguara (S-N), sudeste do Pará: a. modelo 1; b. modelo 2; c. modelo 3

Figure 3 - Two-dimensional gravity models for the Rio Maria-Xinguara section $(\mathrm{S}-\mathrm{N})$ : a. model $1 ; \mathbf{b}$. model $2 ; \mathbf{c}$. model 3

tante assimétrica, com o flanco sul de mergulho suave e aparentando continuidade com a Faixa Serrinha; alcança a profundidade de $4,8 \mathrm{~km}$.
Todos os três modelos são satisfatórios do ponto de vista do ajuste entre os perfis Bouguer medido e calculado. Entretanto, no modelo da figura $3 \mathrm{c}$ foi obtida a melhor coerência com a distribuição das litologias encontradas em mapa. Assim, optouse por considerar esse modelo como o mais adequado para explicar a anomalia Bouguer dos terrenos granito-greenstone ao longo do perfil Rio Maria-Xinguara. Tal resultado foi utilizado na representação da figura 4.

Figura 4 - Modelo geológico bi-dimensional para o perfil Rio Maria-Xinguara, construído com base na correlação de dados geológicos e geofisicos

Figure 4 - Two-dimensional geologic model for the Rio Maria-Xinguara section, based on geological and geophysical correlation

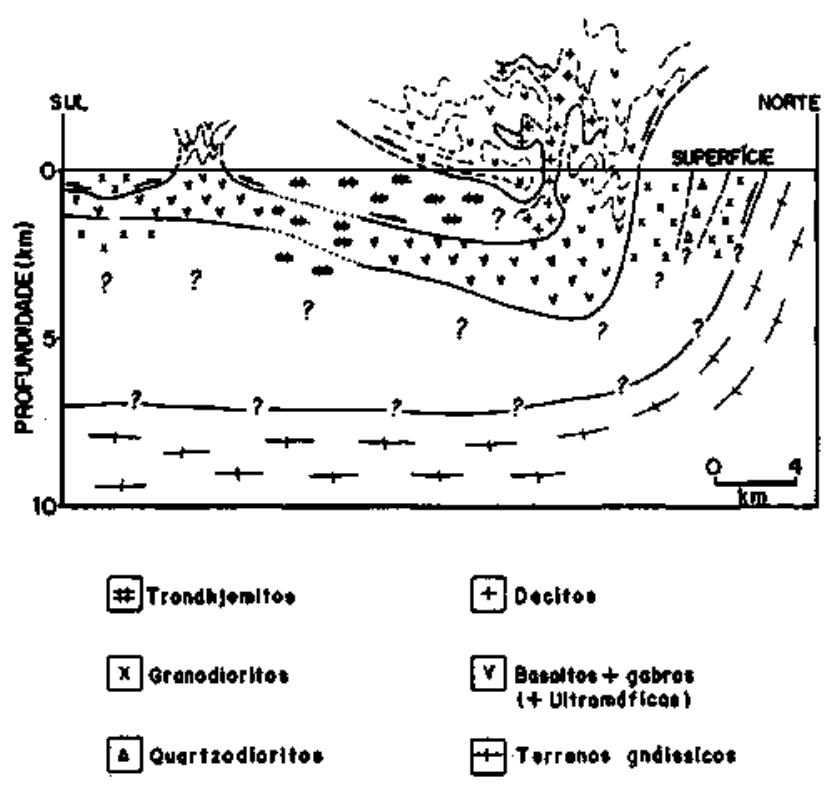

DISCUSSÕES No caso ora estudado, várias das características descritas na literatura se encontram na região de Rio Maria. Por exemplo, ocorrem formas aparentemente tabulares na Faixa Serrinha e sinformais assimétricas na Faixa Identidade, tendo a primeira profundidades menores $(1,8 \mathrm{~km})$ que a segunda $(4,8 \mathrm{~km})$. Adicionalmente, infere-se uma certa continuidade entre o flanco sul da Faixa Identidade e a parte norte da Faixa Serrinha. No caso da Faixa Identidade, o modelo 3 (Fig. $3 c$ ) revela a existência de dois blocos: um mais raso (profundidade de 1,4 km) e outro mais volumoso, que se prolonga em profundidade. Ainda referente a esta faixa, ressalta-se o vale gravimétrico produzido pela presença de metadacitos no seu interior; admite-se que estas rochas ácidas devem ter uma ligação espacial com os trondhjemitos a sul da citada seqüência.

O modelo geológico ilustrado na figura 4 corresponde, naturalmente, a um compromisso entre a geologia de campo e a interpretação gravimétrica da figura 3c. Em acordo com as direções de anomalias Bouguer WNW-ESE ou E-W no sudeste do Pará (Carvalho 1988, 1989), admite-se que as faixas de greenstone belts em foco são alongadas nas mesmas direções. Assim, outros perfis paralelos ao da figura 4 não devem conter modificações substanciais.

A Faixa Serrinha apresenta uma forma diferente da Faixa Identidade e se restringe às porções mais superficiais da crosta. Os granodioritos aflprantes na sua parte sul seriam possivelmente lopolíticos. Com respeito aos trondhjemitos entre Rio Maria e o limite sul da Faixa Identidade, foram interpretadas formas algo tabulares ou mesmo lopolíticas, mergulhando para norte. Para a Faixa Identidade, consideram-se os corpos densos 
delineados na figura $3 \mathrm{c}$ como uma única unidade, seccionada por intrusões hipabissais ácidas (metadacíticas). Por causa do regime de transpressão aplicado à Faixa Identidade (Souza et al 1988), várias fatias dessas rochas supracrustais devem ter sido empurradas sobre os granitóides e posteriormente ezoüdas.

O modelamento atual não fornece respostas sobre o que ocorre abaixo dos greenstone belts. De qualquer modo, admitese como pouco provável a existência de corpos densos em tais locais. O conjunto granito-greenstone estaria restrito aos primeiros cinco quilômetros da crosta. Hipóteses a serem confirmadas com outros estudos são a forma tabular ou megalacolítica e a pequena profundidade dos granodioritos e dos trondhjemitos, conforme proposta da figura 4. Os granodioritos e quartzodioritos a norte da Faixa Identidade permanecem sem interpretação evidente. Por outro lado, os terrenos gnáissicos aflorantes em Xinguara devem representar fatias soerguidas através de falhas lístricas, o que é coerente com a presença, nesta cidade, de dobras em bainha com eixo mergulhando em torno de $35^{\circ}$ para SW.

A Faixa Serrinha tem uma extensão aflorante muito pequena e possui raízes sob os metagranitóides, embora numa escala bem menor (Fig. 2c), enquanto a Faixa Identidade mostra alguma semelhança com a figura $2 \mathrm{~b}$. Considerando que os metagranitóides e os greenstone belts possuem paragêneses de mesmo grau metamórfico (em fácies xisto verde superior e anfibolito inferior), segue-se que não há contrastes de nível erosional que justifiquem o atual padrão geométrico. Logo, as diferentes profundidades dessas supracrustais não podem ser explicadas apenas por falhamentos.

Outras suposições são feitas a partir dos valores médios de gradiente geotérmico e pressões existentes no Arqueano, que seriam de $54^{\circ} \mathrm{C} / \mathrm{km}$ e $4,1 \mathrm{kbar}$, respectivamente (Grambling 1981). Assim, as profundidades teóricas de $1,8 \mathrm{~km} \mathrm{e} \mathrm{4,8} \mathrm{km} \mathrm{das}$ Faixas Serrinha e Identidade corresponderiam aos respectivos incrementos de cerca de $97^{\circ} \mathrm{C}$ e $259^{\circ} \mathrm{C}$. Comparados às fácies metamórficas observadas atualmente, isto significa que as raízes dos greenstones belts estariam em fácies anfibolito superior (Faixa Serrinha) e granulito (Faixa Identidade e gnaisses da região de Xinguara). Admitindo uma relação de 3,3 km para 1 kbar (Grambling 1981), infere-se uma espessura mínima de $18 \mathrm{~km}$ para o bloco crustal granitóides - greenstone belts da região de Rio Maria à época do metamorfismo (final do Arqueano).

Finalmente, a presença de enxames de diques ácidos na área estudada sugere a existência de corpos graníticos em pequena profundidade, que corresponderiam aos tipos anorogênicos descritos a sul de Rio Maria. Nesta hipótese, as anomalias Bouguer aqui descritas e os cálculos de profundidades estariam subestimados.

CONCLUSÕES Mesmo considerando o caráter preliminar da discussão gravimétrica, as três conclusões principais são: 1. as Faixas Serrinha e Identidade possuem formas e profundidades diferentes e se restringem aos primeiros quilômetros da crosta superior; a primeira aparenta ter no máximo $1,8 \mathrm{~km}$ de profundidade, enquanto a segunda atinge $4,8 \mathrm{~km} ; 2$. falhas lístricas nos contatos entre unidades litoestratigráficas propiciariam a intrusão de granitóides com formas tabulares, lacolíticas e lopolíticas, bem como a colocação das rochas gnáissicas; 3. admitem-se diferenças no grau metamórfico nas raízes das unidades enfocadas, alcançando as fácies anfibolito superior (Serrinha) e mesmo granulito (Identidade e gnaisses). A espessura original da crosta na região de Rio Maria seria de no mínimo $18 \mathrm{~km}$.

A realização de um número maior de seções gravimétricas, o prolongamento do perfil tanto para sul de Rio Maria como para norte de Xinguara e a integração com outros métodos geofísicos e com furos de sondagem são necessários para uma melhor definição do que realmente ocorre em profundidade. Isto deverá, por exemplo, informar sobre a delimitação de câmaras magmáticas relativas aos granitóides, às metavulcânicas dos greenstone belts e aos enxames de diques ácidos.

Agradecimentos Os autores são gratos à DOCEGEO (distrito Amazônia), ao CPGG/UFPA e ao DFTF7UFRN pelo apoio nas diversas etapas das pesquisas, ao PADCT-FINEP, CNPq e CAPES pelo financiamento de projetos e bolsas de estudos, a Fernando A.L. Lins (DG/UFRN) e Antônio N.C. Santa Rosa (CPGG/UFPA) por discussões, a Ronald Vieira pelas ilustrações gráficas, a André Luiz Dias de Oliveira pelo auxílio na edição do texto e a dois revisores anônimos da Revista Brasileira de Geociências pelas sugestões.

\section{REFERÊNCIAS BIBLIOGRÁFICAS}

ALMEIDA, F.F.M. 1985. Alguns problemas das relações geológicas entre o Cráton Amazônico e as faixas de dobramentos marginais a leste. In: SIMP. GEOL. CENTRO-OESTE, 2. Goiânia, 1985. Atos... Goiânia, SBG/CO v. 1, p. 3-14.

ALMEIDA, F.F.M ; FERREIRA, F.J.F; CARNEIRO, C.D.R ; STEFANI, EL. 1986. Aspectos evolutivos da geossutura Tocantins-Araguaia. In CONOR. BRAS. GEOL., 34. Goiânia, 1986. Anais... Goiânia, SBG. v. 3, p. 1269-1283.

ALMEIDA, F.F.M.; HASUI, Y.; BRITO NEVES, B.B.; FUCK, R.A. 1977. Províncias estruturais brasileiras. In: SIMP. GEOL. NORDESTE, 8 Campina Grande, 1977. Atas... Campina Grande, SBG/NE. bol. 6, p. 363-391.

AMARAL, G. 1984. Províncias Tapajós e Rio Branco. In: ALMEIDA, F.F.M. \& HASUI, Y. (coords.) O Pré-Cambriano do Brasil. São Paulo, Edgard Blïcher.p. 6-35.

BEER, J.H.; STETTLER, E.H.; BARTON J.M. JR.; REENEN, D.D.; VEARNCOMBE, J.R. 1986. Crustal structure of the Archean granite greenstone terrane in the northern portion of the Kaapval craton. In WORKSHOP ON TECTONIC EVOLUTION OF GREENSTONE BELTS. Houston, 1986. LPI contrib. 584, p. 23-24.

CADY, J.W. 1977. Calculation of Gravity and Magnetic Anomalies Along Profile with end Corrections and Inverse Solutions for Density and Magnetization, Washington, U.S. Geol. Survey. (Open File Report 77-463).

CARVALHO J.S. 1988. Aplicação dos Métodos Gravimétrico e Magnetoméiríco para a Definição do Comportamento Estrutural da Faixa de Dobramentos Araguaia. Belém. 108 p. (Tese de Mestrado, CPGG/UFPA).

CARVALHO, J.S. 1989. Efeitos gravimétricos das seqüências tipo greenstone belts Andorinhas e Serrinha, na região de Rio Maria/Xinguara - sul do Pará. In: CONGR. SOC. BRAS. GEOF., 1. Rio de Janeiro, 1989.Anais.. Rio de Janeiro, SBGf. v. 2, p. 521-526.
CONDE, K. 1981. Archean Greenstone Belts. Amsterdam, Elsevier. 434 p. (Develop. Precamb. Geol. 3).

CRUZ, J.C.R. \& PATVA, R.N. 1988. Levantamento Geofisico - Área Serrinha, Rio Maria, Pará. Belém, CPGG/UFPA. 26 p. (Relatório Curricular da Disciplina Geofísica de Campo).

DALL'AGNOL, R.; VIEIRA, E.A.P.; SÁ, C.A.S.; MEDEIROS, H.; GASTAL, M.C.P.; TEDCEIRA, N.P. 1986. Estado atual do conhecimento sobre as rochas granitóides da porção sul da Amazônia Oriental. Rev. Bras. Geoc., 16(1):11-23

DEPARTAMENTO NACIONAL DA PRODUÇÃO MINERAL 1979. Projeto Geofisica Brasil-Canadá. Mapas de Intensidade Magnética Total. Brasília, DNPM. (mapas diversos na escala 1:100.000, cobrindo a área delimitada pelas coordenadas $51^{\circ} 00^{\prime} \mathrm{W}$ e $48^{\circ} 00^{\prime} \mathrm{W}$ e $5^{\circ} 00^{\prime} \mathrm{S}$ e $12^{\circ} 00^{\prime} \mathrm{S}$ ).

GASTAL, M.C.P.; MACAMBIRA, M.J.B.; MEDEIROS, H.; DALL'AGNOL, R. 1987. Idades e geoquímica isotópica Rb-Sr do Granito Musa e do Granodiorito Rio Maria, Amazônia Oriental. Geochim. Bras., I(2):247-259.

GRAMBLING, J. A. 1981. Pressures and temperatures in Precambrian metamorphic rocks. Earth Planet. Sci. Lett., 53(1):63-68.

HALL, J.M. \& DRURY, M.J. 1989. Greenstone belts and associated granitoids: a CCDP workshop report. Geosci. Can., 16(2):100-103.

HASUI, Y; HARALY, N.I.E.; SCHOBBENHAUS, C. 1984. Elementos geofísicos e geológicos da região Amazônica: subsídios para o modelo geodinâmico. In: SYMP. AMAZ., 2. Manaus, 1984. Anais... Manaus, SBG/NN.v. 1, p. 129-147.

ffIRATA, W.K.; RIGON, J.C.; KADEKARU, K.; CORDEIRO, A.A.C.; MEIRELES, EM. 1982. Geologia regional da Província Mineral de .Carajás. In: SIMP. GEOL. AMAZ., 1. Belém, 1982. Atas... Belém, SBG/ NN.v. 1, p. 100-110

HUHN, S.R.B.; SANTOS, A.B.S.; AMARAL, A.F.; LEDSHAM, E.J.; GOUVEIA, J.L.; MARTINS, L.P.B.; MONTALVÃO, R.M.G.; COSTA, 
V.G. 1988.0 terreno granito-greenstone da região de Rio Maria - sul do Pará. In: CONGR. BRAS. GEOL., 35. Belém, 1988. Anais... Belém, SBG.v. 3, p. 1438-1453.

MACAMBIRA, M.J.B.; LANCELOT, J.R.; LAFON, J.M. 1990. Evolution des granitoides Précambriens de la region de Rio Maria (sud ouest de Amazonie, Brésil) d'apres les données U-Pb, Rb-Sr et K-Ar. In: REUNION DÊS SCIENCES DE LA TERRE, 13. Grenoble, 1990. Resumes... Grenoble, Soc. Géol. Fr. p. 83.

MEDEIROS, H.; GASTAL, M.C.P; DALL'AGNOL, R.; SOUZA, Z.S. 1987. Geology of the Rio Maria area (eastern Amazonian region - Brazil): an example of Archean granite - greenstone terrane intruded by anorogenic granites of middle Proterozoic ages. In: PRECAMBRIAN EVOLUTION OF THE AMAZONIAN REGION. Carajás, 1987. Ext. Abs. Vol... Carajás, 1UGS/UNESCO - IGCP Project 204. p. 97-109.

MONTALVÃO, R.G.M.; TASSINARI, C.C.G.; BEZERRA, P.E.L.; PRADO, P. A. 1984. Geocronologia dos granitóides e gnaisses das regiões de Rio Maria, Fazenda Mata Geral e Rio Itacaiúnas, sul do Pará (Distrito Carajás - Cumaru). In: CONGR. BRAS. GEOL., 33. Rio de Janeiro, 1984. Anais... Rio de Janeiro, SBG. v. 6, p. 2757-2766.

MONTALVÃO, R.G.M.; TASSINARI, C.C.G.; TEIXEIRA, W.; VASCONI, A.V. 1988. Caracterização geocronológica Rb-Sr dos terrenos granodionticos e trondhjemíticos da região de Rio Maria, sul da Serra dos Carajás. In: CONGR. BRAS. GEOL., 35. Belém,1988. Anais... Belém, SBG. v. 3, p. 1478-1487.

RIO DOCE GEOLOGIA E MINERAÇÃO 1988. Revisão litoestratigráfica da Província Mineral de Carajás, Pará. In: CONGR. BRÁS. GEOL., 35. Belém, 1988.Anais... Belém, SBG. p. 11-54

SANTOS, J.O.S. \& LOGUÉRCIO, S.O.C. 1984. A parte meridional do Cráton Amazônico (escudo Brasil-Central) e as bacias do Alto Tapajós e Parecis -
Alto Xingu. In: SCHOBBENHAUS, C.; CAMPOS, D.A.; DERZE, F.R.; ASMUS, H.E. coords. Geologia do Brasil. Brasília, MME/DNPM. p. $93-127$.

SILVA, R.W. \& SÁ, J.H.S. 1982. Feiç̃es geológicas e magnetométricas da região do Baixo Araguaia. In: SIMP. GEOL. AMAZ., 1. Belém, 1982. Atas... Belém, SBG/NN. v. 1, p. 259-269.

SOUZA, Z.S.; MEDEIROS, H.; ALTHOFF, F.J.; DALL'AGNOL, R. 1990. Geologia do terreno granito-greenstone arqueano da Região de Rio Maria sudeste do Pará. In: CONGR. BRAS. GEOL., 36. Natal, 1990. Anais.. Natal, SBG. v. 6, p. 2913-2928.

SOUZA, Z.S.; SANTOS, A.B.; LEDSHAM, E.J.; HUHN, S.R.B.; COSTA, V.G. 1988. Feições geológicas e estruturais do greenstone belt de Identidade, região de Xinguara - Rio Maria, sul do Pará. In: CONGR. BRAS. GEOL., 35. Belém, 1988. Anais... Belém, SBG. v. 3, p. 1453-1467.

TALWANI, M.; WORZEL, J.L.; LANDISMAN, M. 1959. Rapid gravity computations for two-dimensional bodies with application to the Mendocino submarine fracture zone. J. Geophys. Res., 64:49-59.

THOMAS, M.D.; LOSIER, L.; THURSTON, P.C.; GUPTA, V.K.; GIBB, R. A GRIEVE, R.A.F. 1986. Geophysical Characteristics and crustal structure of the greenstone terranes, Canadian shield. In: WORKSHOP ON THE TECTONIC EVOLUTION OF GREENSTONE BELTS. Houston, 1986. LPI contrib. 584, p. 123-125.

MANUSCRITO A695

Recebido em I de março de 1991 Revisão do autor em 18 de dezembro de 1991 Revisão aceita em 20 de dezembro de 1991 